\title{
A UNIFIED SPACE FOR MAPPINGS
}

\section{BX \\ G. T. WHYBURN}

1. Introduction. Although as a concept in its own right the compact mapping is of fairly recent vintage, it is already familiar to many users of the mathematical language of today as it merely isolates and formalizes the familiar fact that, when a mapping operates on a compact space, the inverse image of every compact set is compact. Thus in general a mapping is compact $[1 ; 2]$ when it has this property, whether the domain space is compact or not. This notion has already proven fruitful and offers promise of further useful exploitation due largely to the fact that a compact mapping retains in a more general setting so much of the character of a mapping operating on a compact space. Thus a considerable advantage accrues when problems concerning mappings on noncompact spaces can be reduced to a form allying them as closely as possible with compact mappings.

In view of the fact that, as shown by Varnštein [2], any closed mapping has a partial mapping which is compact and has the same image space, and in view also of the usual duality relationship between open and closed mappings, it has seemed to the author that an arbitrary open mapping should be related in some analogous way to a compact mapping. One might anticipate that if the domain space were suitably augmented the mapping could be so extended as to become compact, so that the given mapping would be a partial mapping of a compact one. In this paper it will be shown that this can in fact be done and, indeed, for all mappings rather than for just open ones. For it turns out that in a suitably constructed space, unifying both the domain and the range spaces, a compact mapping can be defined which is topologically equivalent to the given one on the prototype of the original domain space. Thus we show that any mapping from one Hausdorff( $\left.{ }^{1}\right)$ space to another is topologically equivalent to a partial mapping of a compact mapping (actually a retraction) in the unified space. The unified space is separable and metrizable when the given spaces are locally compact, separable and metric; and it is in this setting that the chief usefulness of the new viewpoint and result will likely be found.

2. The unified space. Open sets. The transfer mappings. Given topological spaces $X$ and $Y$ with open set topologies and a mapping $f(X)=Y$, we define a new space, called the unified space,

Presented to the Society, September 5, 1952; received by the editors March 20, 1952.

(1) The author is indebted to J. L. Kelley for the suggestion that this be done for Hausdorff spaces rather than separable metric spaces. 


$$
Z=X^{\prime}+Y^{\prime}
$$

consisting of a point $x^{\prime}=h(x)$ for each $x \in X$ and a point $y^{\prime}=k(y)$ for each $y \in Y$ so that $h(X)=X^{\prime}$ and $k(Y)=Y^{\prime}$ are 1-1 and $X^{\prime} \cdot Y^{\prime}=0$ (the empty set). A subset $Q$ of $Z$ is defined to be open provided

$$
\begin{aligned}
& h^{-1}\left(Q \cdot X^{\prime}\right) \text { is open in } X, \\
& k^{-1}\left(Q \cdot Y^{\prime}\right) \text { is open in } Y,
\end{aligned}
$$

and

(iii) for any compact( $\left.{ }^{2}\right)$ set $K \subset k^{-1}\left(Q \cdot Y^{\prime}\right), f^{-1}(K) \cdot\left[X-h^{-1}\left(Q \cdot X^{\prime}\right)\right]$ is compact.

As usual a set $F$ in $Z$ is defined to be closed provided $Z-F$ is open. A subset $V$ of $Y^{\prime}$ is said to be open in $Y^{\prime}$ if and only if it is of the form $V=Q \cdot Y^{\prime}$ where $Q$ is open in $Z$ and, similarly, a set $U$ in $X^{\prime}$ is open in $X^{\prime}$ provided it is of the form $U=Q \cdot X^{\prime}$ with $Q$ open in $Z$. When the term "open" without qualification is used in connection with a subset of $Z$ it is meant to refer to openness in $Z$ as defined above.

We note the following easy conclusions concerning open sets in $Z$.

(a) If $Q$ is open so also is $Q \cdot X^{\prime}$. In particular, $X^{\prime}$ is open.

For (ii) and (iii) follow at once from the fact that $Y^{\prime} \cdot Q \cdot X^{\prime}$ is empty.

(b) $A$ subset $E$ of $X^{\prime}$ is open in $X^{\prime}$ if and only if it is open in $Z$.

The "if" is trivial and the "only if" follows from (a).

(c) For any open set $U$ in $X, h(U)$ is open.

For again $h(U) \cdot Y^{\prime}$ is empty, giving (ii) and (iii).

(d) If $V$ is any open set in $Y, X^{\prime}+k(V)$ is open.

For if $Q=X^{\prime}+k(V)$, (i) and (ii) are satisfied because $h^{-1}\left(Q \cdot X^{\prime}\right)=X$ and $k^{-1}\left(Q \cdot Y^{\prime}\right)=V$; and (iii) follows from the fact that $X-h^{-1}\left(Q \cdot X^{\prime}\right)$ is empty.

(e) For any closed set $K \subset Y, Z-k(K)$ is open so that $k(K)$ is closed.

This follows from (d), taking $V=Y-K$.

These conclusions lead at once to the

THEOREM. $h$ is an open mapping of $X$ into $Z$ and $k$ is a closed mapping of $Y$ into $Z$. Thus $h$ and $k$ are homeomorphisms.

Continuity of $h$ and $k$ results directly from (i) and (ii) respectively. From (c) and (e) we have that $h$ is open and that $k$ is closed. Since $h$ and $k$ are 1-1 they are therefore homeomorphisms. They will be called the transfer mappings.

3. Retraction onto $Y^{\prime}$. For each $x^{\prime} \in X^{\prime}$ define $r\left(x^{\prime}\right)=k f h^{-1}\left(x^{\prime}\right)$ and for $y^{\prime} \in Y^{\prime}$ define $r\left(y^{\prime}\right)=y^{\prime}$.

THEOREM. $r$ is continuous and hence is a retraction of $Z$ into $Y^{\prime}$.

(2) The term "compact" is used throughout this paper in the sense of "bicompact," i.e., a set is compact if each of its open coverings is reducible to a finite covering. 
Proof. Let $U$ be any open subset of $Y^{\prime}$. We have to show that $r^{-1}(U)$ is open in $Z$. Let $Q=r^{-1}(U)$. We have

$$
Q=U+h f^{-1} k^{-1}(U) .
$$

Then $Q$ satisfies (i) since $Q \cdot X^{\prime}=h f^{-1} k^{-1}(U)$ so that $h^{-1}\left(Q \cdot X^{\prime}\right)=f^{-1} k^{-1}(U)$, which is open by continuity of $k$ and $f$; and (ii) holds since $Q \cdot Y^{\prime}=U$ so that $k^{-1}\left(Q \cdot Y^{\prime}\right)=k^{-1}(U)$ which is open by continuity of $k$. Also $Q$ satisfies (iii), because any compact set $K \subset k^{-1}\left(Q \cdot Y^{\prime}\right)=k^{-1}(U)$ has $f^{-1}(K) \subset h^{-1}\left(Q \cdot X^{\prime}\right)$ $=f^{-1} k^{-1}(U)$. Thus $Q$ is open in $Z$.

4. Open set axioms satisfied in the unified space. We now assume $X$ and $Y$ to be Hausdorff spaces, i.e., topological spaces in which any two distinct points have disjoint open set neighborhoods. On this basis we show that $Z$ is a topological space with an open set topology. Also $Z$ satisfies the weaker separation axiom that "the complement of a point is open" although in general not the Hausdorff separation axiom.

(4.1) The empty set and the whole space $Z$ are open sets.

Proof. If $Q$ is empty, so are $h^{-1}\left(Q \cdot X^{\prime}\right)$ and $h^{-1}\left(Q \cdot Y^{\prime}\right)$ so that (i) and (ii) are satisfied. Also any $K \subset k^{-1}\left(Q \cdot Y^{\prime}\right)$ is empty so that $f^{-1}(K)$ is empty and thus (iii) holds. If $Q=Z, h^{-1}\left(Q \cdot X^{\prime}\right)=X$ and $k^{-1}\left(Q \cdot Y^{\prime}\right)=Y$ so that (i) and (ii) are satisfied, whereas $X-h^{-1}\left(Q \cdot X^{\prime}\right)$ is empty so that (iii) holds.

LEMma. In a Hausdorff space, any compact set $K \subset \sum_{1}^{n} G_{i}$, where each $G_{i}$ is open, admits a representation $K=\sum_{1}^{n} K_{i}$ where each $K_{i}$ is compact and $K_{i} \subset G_{i}, i=1, \cdots, n$.

Proof. By induction on $n$. The case $n=1$ is trivial. Assume true for $n<k$. Given $K \subset \sum_{1}^{k} G_{i}$, let $A=K-K \cdot \sum_{1}^{k-1} G_{i}$ and $B=K-K \cdot G_{k}$. Since $A$ and $B$ are compact and disjoint, there exists an open set $V$ satisfying $A \subset V \subset G_{k}$ and $\bar{V} \cdot B=0$ so that $K \cdot \bar{V} \subset G_{k}$. Define $K_{k}=K \cdot \bar{V}$ and $K^{\prime}=K-K \cdot V$. Now since $K^{\prime}$ is compact and $K^{\prime} \subset \sum_{1}^{k-1} G_{i}$, by the induction hypothesis we have $K^{\prime}=\sum_{1}^{k-1} K_{i}$ where $K_{i}$ is compact and $K_{i} \subset G_{i}, i=1, \cdots, k-1$. This gives $K=\sum_{1}^{k} K_{i}$ with $K_{i}$ compact and $K_{i} \subset G_{i}, i=1, \cdots, k$, and thus proves our lemma.

(4.2) The union of any collection of open sets is an open set.

Proof. Let $U=\sum Q$, where each set $Q$ is open in $Z$. Then since $h^{-1}\left(U \cdot X^{\prime}\right)$ $=\sum h^{-1}\left(Q \cdot X^{\prime}\right)$ and each set $h^{-1}\left(Q \cdot X^{\prime}\right)$ is open, it follows that (i) is satisfied. Similarly, (ii) is satisfied. Now let $K$ be a compact set lying in $k^{-1}\left(U \cdot Y^{\prime}\right)$ $=\sum k^{-1}\left(Q \cdot Y^{\prime}\right)$. Since each set $k^{-1}\left(Q \cdot Y^{\prime}\right)$ is open in $Y$, there is a finite sequence of the sets $Q$, say $Q_{1}, \cdots, Q_{n}$, such that

$$
K \subset \sum_{1}^{n} k^{-1}\left(Q_{i} \cdot Y^{\prime}\right)
$$

By the lemma, this gives 


$$
K=\sum_{1}^{n} K_{i}, \text { where each } K_{i} \text { is compact and } K_{i} \subset k^{-1}\left(Q_{i} \cdot Y^{\prime}\right) .
$$

We have

$$
\begin{aligned}
f^{-1}(K)\left[X-h^{-1}\left(U \cdot X^{\prime}\right)\right] & =\sum_{1}^{n} f^{-1}\left(K_{i}\right)\left[X-h^{-1}\left(U \cdot X^{\prime}\right)\right] \\
& \subset \sum_{i=1}^{n} f^{-1}\left(K_{i}\right) \cdot\left[X-h^{-1}\left(X^{\prime} \cdot \sum_{1}^{n} Q_{j}\right)\right] \\
& \subset \sum_{i=1}^{n} f^{-1}\left(K_{i}\right)\left[X-h^{-1}\left(X^{\prime} \cdot Q_{i}\right)\right] .
\end{aligned}
$$

The final set on the right is compact because each of the summands is compact by (iii) and the openness of $Q_{i}$. Thus the set on the left is compact since it is closed. Accordingly (iii) is satisfied by $U$ and $U$ is open.

We remark that $a$ set in $Z$ is closed if and only if it contains all of its limit points. This is now clear because, in any space, the proposition that every set containing all its limit points has an open complement is equivalent to the proposition that the union of any collection of open sets is open.

(4.3) The intersection of two open sets is open.

Proof. Let $Q_{1}$ and $Q_{2}$ be open. Then since $h^{-1}\left(Q_{1} \cdot Q_{2} \cdot X^{\prime}\right)=h^{-1}\left(Q_{1} \cdot X^{\prime}\right)$ $\cdot h^{-1}\left(Q_{2} \cdot X^{\prime}\right)$, it follows that (i) is satisfied. Similarly (ii) is satisfied. Let $K$ be a compact set contained in $k^{-1}\left(Q_{1} \cdot Q_{2} \cdot Y^{\prime}\right)$. Then

$$
\begin{aligned}
& f^{-1}(K)\left[X-h^{-1}\left(Q_{1} \cdot Q_{2} \cdot X^{\prime}\right)\right] \\
&=f^{-1}(K)\left[X-h^{-1}\left(Q_{1} \cdot X^{\prime}\right) \cdot h^{-1}\left(Q_{2} \cdot X^{\prime}\right)\right] \\
&=f^{-1}(K)\left[X-h^{-1}\left(Q_{1} \cdot X^{\prime}\right)+X-h^{-1}\left(Q_{2} \cdot X^{\prime}\right)\right] \\
&=f^{-1}(K)\left[X-h^{-1}\left(Q_{1} \cdot X^{\prime}\right)\right]+f^{-1}(K)\left[X-h^{-1}\left(Q_{2} \cdot X^{\prime}\right)\right] .
\end{aligned}
$$

Since each summand on the right is compact it follows that the set on the left is compact and thus (iii) is satisfied by $Q_{1} \cdot Q_{2}$.

(4.4) For any $p \in Z, Z-p$ is open.

Proof. If $Z-p=Q$, the sets $h^{-1}\left(Q \cdot X^{\prime}\right)$ and $h^{-1}\left(Q \cdot Y^{\prime}\right)$ are the same as $X$ and $Y$ or these spaces reduced by a single point. Thus (i) and (ii) hold. Also the set $X-h^{-1}\left(Q \cdot X^{\prime}\right)$ contains at most one point, so that (iii) holds for any $K$.

(4.41) Corollary. For any compact set $N$ in $X, Z-h(N)$ is open.

For (i) and (ii) follow as in (4.4); and the set in (iii) becomes $f^{-1}(K) \cdot N$ and hence is compact.

5. Compactness of $r$. Openness.

(5.1) TheOREM. The retraction $r$ is a compact mapping, i.e., for any compact set $K^{\prime}$ in $Y^{\prime}, r^{-1}\left(K^{\prime}\right)$ is compact. 
Proof. Since $K=k^{-1}\left(K^{\prime}\right)$ is compact so that $Y-K$ is open, it follows by $\S 2$, (d), that $X^{\prime}+Y^{\prime}-K^{\prime}=Z-K^{\prime}$ is open so that $K^{\prime}$ is closed. Hence $r^{-1}\left(K^{\prime}\right)$ is closed. Let $[G]$ be any open covering of $r^{-1}\left(K^{\prime}\right)$. Since $K^{\prime}$ is compact, a finite union $U=\sum_{i=1}^{n} G_{i}$ of sets of $[G]$ contains $K^{\prime}$. Let

$$
E=r^{-1}\left(K^{\prime}\right)-U r^{-1}\left(K^{\prime}\right) .
$$

Then $E$ is closed and $E \subset X^{\prime}$. Thus $Q=Z-E$ is open and contains $K^{\prime}$. Since $K \subset k^{-1}\left(Q \cdot Y^{\prime}\right)$, it follows by (iii) that the set

$$
f^{-1}(K) \cdot\left[X-h^{-1}\left(Q \cdot X^{\prime}\right)\right]
$$

is compact. Since $h^{-1}(E)$ is closed and is contained in this set, $h^{-1}(E)$ is compact. Therefore $E$ is compact and hence is contained in a finite union $V$ $=\sum_{n+1}^{m} G_{i}$ of sets of $[G]$. This gives $r^{-1}\left(K^{\prime}\right) \subset U+V=\sum_{1}^{m} G_{i}$.

(5.11) Corollary. If $Y$ is locally compact, so also is $Z$. If $Y$ is compact so also is $Z$.

Thus for any space $X$, the corresponding space $Z$ provides a compactification or local compactification of $X$ for every mapping of $X$ onto a compact $Y$ or a locally compact $Y$ respectively.

(5.2) TheOREM. If $f$ is an open mapping, so also is $r$.

Proof. Let $Q$ be any open set in $Z$. By definition of $r$ we have

$$
r(Q)=k f h^{-1}\left(Q \cdot X^{\prime}\right)+Q \cdot Y^{\prime} ;
$$

and since each of the sets on the right is open in $Y^{\prime}$ so also is their union.

6. Regularity. If $X$ and $Y$ are locally compact, $Z$ is regular.

Proof. Let $p \in Z$ and let $Q$ be any open set in $Z$ containing $p$.

If $p \in X^{\prime}, X^{\prime} \cdot Q$ is open and contains $p$. Thus $h^{-1}\left(X^{\prime} \cdot Q\right)$ contains an open set $V$ containing $h^{-1}(p)$ and such that $\bar{V}$ is compact and lies in $h^{-1}\left(X^{\prime} \cdot Q\right)$. By $\$ 1,(c), h(V)$ is open in $Z$ and, by (4.41), $h(\bar{V})$ is closed in $Z$; and since $h(\bar{V}) \supset h(V)$ we have

$$
p \subset h(V) \subset \overline{h(V)} \subset h(\bar{V}) \subset X^{\prime} \cdot Q .
$$

If $p \in Y^{\prime}$, let $U$ be an open set in $Y$ such that $\bar{U}$ is compact and satisfying

$$
k^{-1}(p) \subset U \subset \bar{U} \subset k^{-1}\left(Q \cdot Y^{\prime}\right) .
$$

Then the set

$$
K=f^{-1}(\bar{U})\left[X-h^{-1}\left(Q \cdot X^{\prime}\right)\right]
$$

is compact. Thus since $X$ is locally compact there exists an open set $G$ in $X$ containing $K$ such that $\bar{G}$ is compact. We define

$$
V=Q \cdot[Z-h(\bar{G})] \cdot r^{-1} k(U) .
$$


Then $V$ is open in $Z$ since each of the three sets on the right is open in $Z$. Obviously $V$ contains $p$ and is contained in $Q$. Let $x \in F_{r}(V)=\bar{V}-V$. Then

$$
x \in r^{-1} k(\bar{U})=r^{-1} \overline{k(U)},
$$

since this latter set is closed and contains $r^{-1} k(U)$ which in turn contains $V$. Now if $x \in X^{\prime}-Q \cdot X^{\prime}$, we would have $h^{-1}(x) \in K \subset G$, because $k f h^{-1}(x)=r(x)$ must be in $k(\bar{U})$ so that $f h^{-1}(x)$ must be in $\bar{U}$ and hence $h^{-1}(x)$ is in $f^{-1}(\bar{U})$ and not in $h^{-1}\left(Q \cdot X^{\prime}\right)$, i.e. $h^{-1}(x) \in K$. However, this is impossible as $V \subset Z$ $-h(\bar{G})$. On the other hand, if $x \in Y^{\prime}$, we have $r(x)=x$ and thus $x \in k(\bar{U}) \subset Q$. Thus in any case $x \in Q$ so that $\bar{V} \subset Q$.

In view of (5.11) it is clear that regularity of $Z$ is not to be expected without additional restrictions on $X$ since, for example, if $X$ is an arbitrary subset of a Euclidean or of Hilbert space and $f$ is a projection of $X$ onto a line, the resulting unified space $Z$ would be locally compact and yet it contains an open set homeomorphic with $X$. Here it will be noted that if, for example, $X$ and $Y$ are in $E_{2}$ and $X$ contains a dense subset of a line perpendicular to $Y$ but contains no interval of this line, the unified space $Z$ does not even satisfy the Hausdorff separation axiom. Indeed we have the

THEOREM. If $Y$ is locally compact, then $Z$ is regular if and only if $X$ is locally compact.

Proof. If $X$ is locally compact, $Z$ is regular by the preceding theorem. On the other hand, by (5.11), $Z$ is locally compact; and since $X^{\prime}$ is open in $Z$, this implies that $X^{\prime}$ is locally compact provided $Z$ is regular. Thus if $Z$ is regular, $X^{\prime}$ is locally compact and hence so also is $X$ as $h(X)=X^{\prime}$ is a homeomorphism.

7. Separability. If $X$ and $Y$ are locally compact and perfectly separable, $Z$ is perfectly separable.

Proof. Choose a countable base $R_{1}, R_{2}, \cdots$, of open sets in $Y$ with each $\bar{R}_{n}$ compact and a countable base $Q_{1}, Q_{2}, \cdots$ in $X$. Since $f^{-1}\left(\bar{R}_{n}\right)$ is closed and $X$ is locally compact we can write

$$
f^{-1}\left(\bar{R}^{n}\right)=\sum_{i=1}^{\infty} K_{i}^{n}
$$

where each set $K_{i}^{n}$ is compact and

$$
K_{i}^{n} \subset \operatorname{int}\left(K_{2}^{n}\right) \subset K_{2}^{n} \subset \operatorname{int}\left(K_{3}^{n}\right) \subset \ldots
$$

where "int" means interior relative to $f^{-1}\left(\bar{R}^{n}\right)$. Define

$$
\begin{aligned}
S_{i}^{n} & =r^{-1} k\left(R_{n}\right)-h\left(K_{i}^{n}\right) \\
& =r^{-1} k\left(R_{n}\right) \cdot\left[Z-h\left(K_{i}^{n}\right)\right], \quad i, n=1,2, \cdots .
\end{aligned}
$$


Then each $S_{t}^{n}$ is open since each of the two sets on the right in the last equation is open. We shall show that the sets $\left(S_{i}^{n}\right)$ together with the sets $\left[h\left(Q_{n}\right)\right]$ form a countable basis in $Z$.

To this end let $p \in Z$ and let $Q$ be any open set in $Z$ containing $p$. If $p \in X^{\prime}$, then since $Q \cdot X^{\prime}$ is open, there exists an $n$ such that $h^{-1}(p) \in Q_{n} \subset h^{-1}\left(Q \cdot X^{\prime}\right)$ and thus so that $p \in h\left(Q_{n}\right) \subset Q \cdot X^{\prime} \subset Q$. On the other hand, if $p \in Y^{\prime}$, let us choose an $n$ so that

$$
k^{-1}(p) \subset R_{n} \subset \bar{R}_{n} \subset k^{-1}\left(Q \cdot Y^{\prime}\right)
$$

and let

$$
K=f^{-1}\left(\bar{R}_{n}\right) \cdot\left[X-h^{-1}\left(Q \cdot X^{\prime}\right)\right] .
$$

Then $K$ is compact, and since $K \subset f^{-1}\left(\bar{R}_{n}\right)=\sum_{i=1}^{\infty} K_{i}^{n}$, there exists an $m$ such that $K \subset K_{m}^{n}$. This gives

$$
h\left(K_{m}^{n}\right) \supset h f^{-1}\left(\bar{R}_{n}\right) \cdot\left[X^{\prime}-Q \cdot X^{\prime}\right]
$$

so that

$$
S_{m}^{n}=r^{-1} k\left(R_{n}\right)-h\left(K_{m}^{n}\right)=h f^{-1}\left(R_{n}\right)-h\left(K_{m}^{n}\right)+k\left(R_{n}\right) \subset Q .
$$

8. Conclusion. The results in the preceeding sections give at once the

Theorem. For any mapping $f(X)=Y$ where $X$ and $Y$ are locally compact, separable and metric, the unified space $Z$ is separable and metric and $f$ is topologically equivalent to $r \mid X^{\prime}$ where $r$ is a compact retraction of $X$ into $Y$.

\section{BIBLIOGRAPHY}

1. G. T. Whyburn, Memoirs of the American Mathematical Society, no. 1, 1950. See also Duke Math. J. vol. 17 (1950) pp. 69-74.

2. I. A. VaY̌nšteInn, Doklady Akademii Nauk SSSR. N.S. vol. 57 (1947) pp. 319-321.

UNIVERSITY OF VIRGINIA,

Charlottesville, Va. 